\title{
Wide-Angle Localization of Intraocular Devices from Focus
}

\author{
Christos Bergeles, Kamran Shamaei, Jake J. Abbott, and Bradley J. Nelson
}

\begin{abstract}
Future retinal therapies will be partially automated in order to increase the surgeons' ability to operate near the sensitive structure of the human eye retina. Untethered robotic devices that achieve the desired precision have been proposed, but require localization information for their control. Since the interior of the human eye is externally observable, vision can be used for localization. Previously, a focus-based paraxial localization algorithm using a Mechatronic Vitreoretinal Ophthalmoscope (MVO) was proposed and evaluated by the authors. In this paper, the first algorithm for wideangle intraocular localization is presented. The effectiveness of this new localization approach is demonstrated by experiments using a model eye and a customized MVO, and there is clear improvement over previously reported results.
\end{abstract}

\section{INTRODUCTION}

In order to successfully perform operations near the sensitive structure of the human retina, increased dexterity that is often beyond the limits of human surgeons is required. To increase the success of retinal surgeries, a variety of robotic assistants have been proposed. In [1]-[3] tethered robotic systems for retinal treatment with increased accuracy are presented, and in [4], an untethered biomedical microrobot that can freely move in the posterior of the eye is proposed.

To accurately control intraocular devices, knowledge of their position in the interior of the eye is generally required. For tethered robotic systems, this information can be extracted from their internal sensors. However, for untethered devices such as [4], the necessary localization information must be provided externally. Since the interior of the human eye is observable, images and vision can be used for the localization of untethered intraocular agents.

For successful intraocular imaging additional ophthalmic lenses must be used [5]. These lenses, together with the optics of the human eye, alter the formation of images and make widely used calibration-based pose estimation methods (e.g. [6]) inappropriate. Thus, in order to extract position information of intraocular agents from images, other approaches must be considered.

Our work is motivated from the magnetically steered intraocular microrobot presented in [4]. For successful control, knowledge of the position of the device within the magnetic field is necessary [7], [8]. In order to avoid conflicts with the magnetic steering system (or any other steering system), we perform imaging and localizing with a stationary system.

This work was supported by the NCCR Co-Me of the Swiss National Science Foundation.

The authors are with the Institute of Robotics and Intelligent Systems, ETH Zurich, 8092 Zurich, Switzerland \{cbergeles, kamrans, bnelson\} eethz.ch. Jake J. Abbott is now with the Department of Mechanical Engineering, University of Utah, Salt Lake City, Utah 84112 jake.abbotteutah.edu

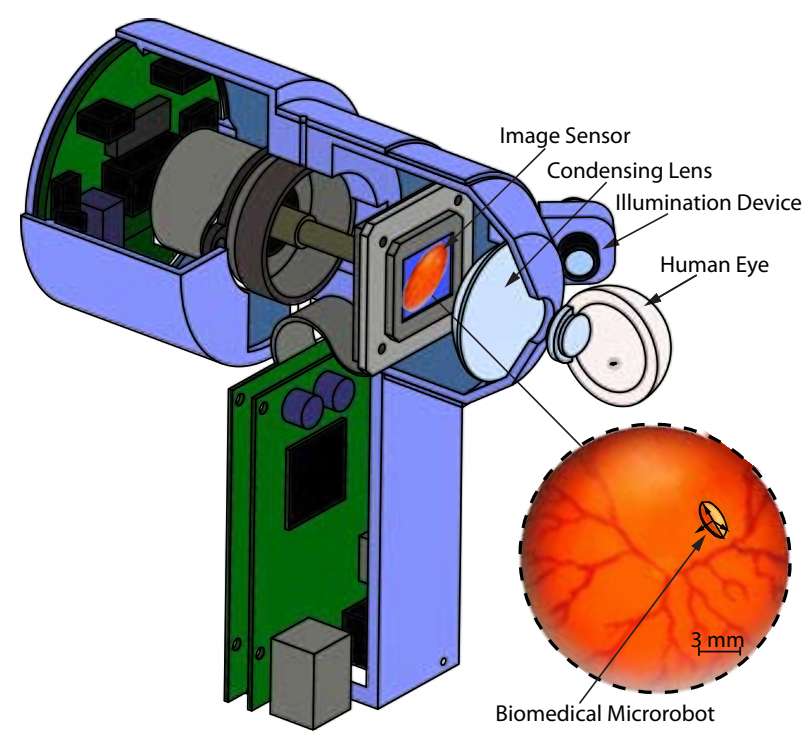

Fig. 1. Illustration of the Mechatronic Vitreoretinal Ophthalmoscope for intraocular device localization.

In [9] the effects of the human eye optics and different ophthalmic observation methods on the image formation are quantified. The conclusion is that indirect ophthalmoscopy is an appropriate method for intraocular imaging and localization, and successful focus-based paraxial localization is demonstrated. In this paper, we use a custom Mechatronic Vitreoretinal Ophthalmoscope (MVO) (Fig. 1), and propose the first wide-angle intraocular localization algorithm.

The proposed algorithm can be applied for arbitrary optical systems, and is based on extracting depth information from focus [10]. Depth-from-focus techniques require information only on the optical system, and can thus be used in order to localize unknown objects. As a result, the described method need not be considered only in the scope of robotic agent localization, but can be used to estimate the position of foreign intraocular bodies in general. Focus information is also used in [11] to visually servo intraocular microrobots, however, the optics of the eye are not properly considered.

In the following section, the concept of indirect ophthalmoscopy and its potential in imaging and localizing intraocular agents is introduced. In Sec. III the theory of wide-angle intraocular localization is discussed, and in Sec. IV a complete experimental validation is presented. An appropriate MVO is designed and prototyped, and wideangle localization is performed in a model eye. Section V reports our conclusions.

Throughout this paper, the angles and the field-of-view are measured from the pupil and around the optical axis. 


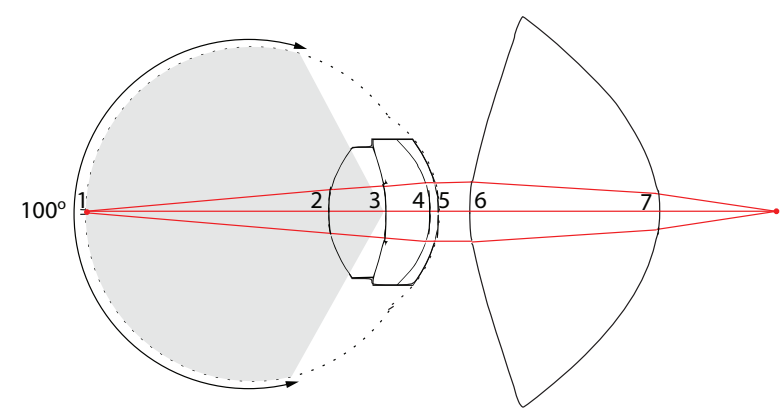

(a)

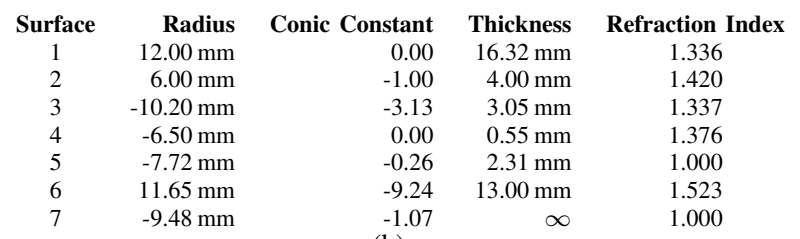

(b)

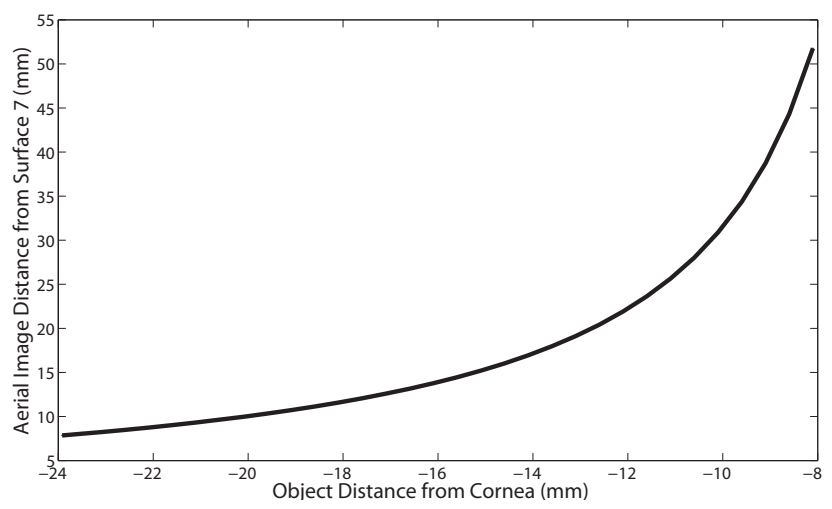

(c)

Fig. 2. (a) Navarro's schematic eye [12] with condensing lens [13]. (b) Optical parameters of the system. (c) Aerial image position versus intraocular object position for indirect ophthalmoscopy.

Our results are based on Navarro's schematic eye [12]. A schematic eye is an optical model of the human eye that is based on averaged biometric data, and which explains the optics of the eye within a certain area of validity. The area of validity of the Navarro eye is $\sim 70^{\circ}$. We will consider only relaxed (or paralyzed) eyes that focus incoming rays on the retina. We also assume that we have sufficient illumination to acquire our images.

\section{INDIRECT OPHTHALMOSCOPY FOR IMAGING AND LOCALIZING}

Indirect ophthalmoscopy uses condensing lenses that create a flat aerial image of the spherical surface of the retina. Contrary to other ophthalmoscopy methods, indirect ophthalmoscopy allows a wide field-of-view to be observed. From simulations in OSLO (optical lens design software) for a system composed of Navarro's schematic eye equipped with a condensing lens (Fig. 2(a)), the aerial image position versus the on-axis object position can be estimated (Fig. 2(c)). The results indicate that if the created aerial image is directly captured by an image sensor with a shallow depthof-field, not only a high field-of-view is possible, but also accurate focus-based localization [9]. As a result, indirect ophthalmoscopy is our method of choice.

\section{WIDE-ANGLE INTRAOCULAR LOCALIZATION}

In [9], an algorithm for intraocular localization based on paraxial approximations was presented. This algorithm was successful for a field-of-view of $\sim 20^{\circ}$. The reason for inaccuracy at higher angles is two-fold: paraxial approximations rely on small-angle assumptions, and these approximations assume plane-to-plane image mappings. As previously stated though, it is the spherical surface of the retina that is projected on a flat aerial image.

One expects that a moving sensor will focus on different surfaces inside the eye; we call these surfaces isofocus surfaces. Moreover, locations inside the eye will correspond to pixels on a moving sensor in a way that differs from the perspective projection model; the locus of intraocular points that are imaged on the same pixel is called an isopixel curve.

The existence of isofocus surfaces and isopixel curves is certain, although their locations are dependent on the MVO and the individual eye. For a well-modeled optical system based on biometric measurements [14], [15], the surfaces and curves can be accurately computed offline. In theory there are an infinite number of isofocus surfaces and isopixel curves, but in practice there will be a finite number due to the resolution of sensor movement and pixel size, respectively.

We can estimate the isofocus surfaces and the isopixel curves with exact raytracing for Navarro's eye. Due to the rotational symmetry of the system, we examine the 2D case. For a grid of points inside the eye, a fan of rays is traced back to the sensor. We position the sensor plane so that the spot created by this rayfan is minimized (i.e. the image is in focus). The $2 \mathrm{D}$ coordinates on the sensor plane where the rayfan is focused specify the pixel coordinates on the image. With the calculated information we create the isofocus surfaces and isopixel curves. The results within the area of validity of the Navarro eye can be seen in Fig. 3. The position of an intraocular point can be estimated from the intersection of its isopixel curve (determined from its pixel coordinates on the in-focus image) with its isofocus surface (determined from the displacement of the sensor with respect to the condensing lens).

From the density of the isofocus surfaces for uniform sensor steps in Fig. 3, we understand that the expected depth resolution is higher for regions far from the retina. This is expected from Fig. 2(c) as well. From the isopixel curves we realize that the formed image is inverted, and from their slope we deduce that the intraocular magnification of an intraocular object increases farther from the retina. As a result, we conclude that both spatial and lateral resolutions increase for positions farther from the retina.

In order to be able to perform intraocular localization unambiguously, the parameters of the isofocus surfaces and isopixel curves should be injective functions of the sensor position and of the pixel coordinates, respectively.

The isofocus surfaces result from the optics of a rotationally symmetric and aligned system composed of conic 


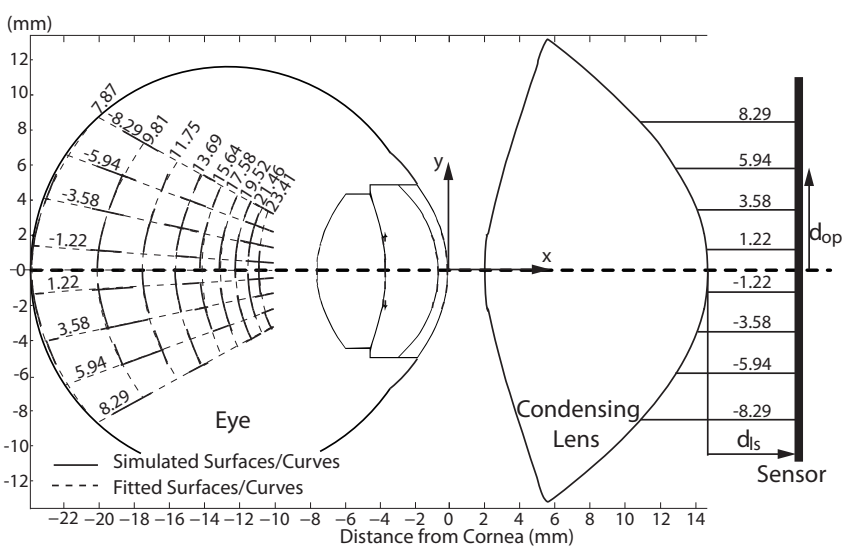

Fig. 3. Simulation and fits for the isofocus surfaces and isopixel curves of the system of Fig. 2(a). The different isofocus surfaces correspond to the distance from the lens to the sensor $\left(d_{l s}\right)$, for uniform sensor steps of $\sim 1.95 \mathrm{~mm}$. The isopixel curves correspond to pixel distances from the optical axis $\left(d_{o p}\right)$, for uniform steps of $\sim 2.25 \mathrm{~mm}$. The simulated surfaces and the fitted ones are hard to distinguish.
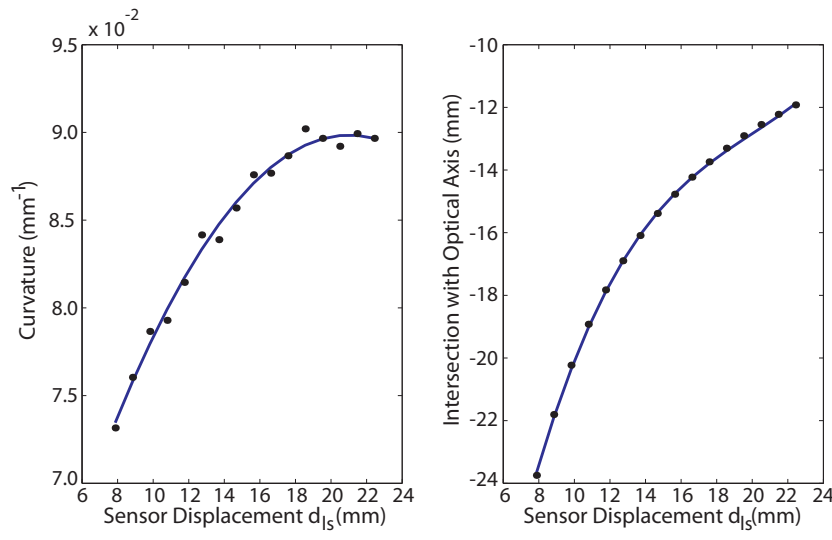

(a)
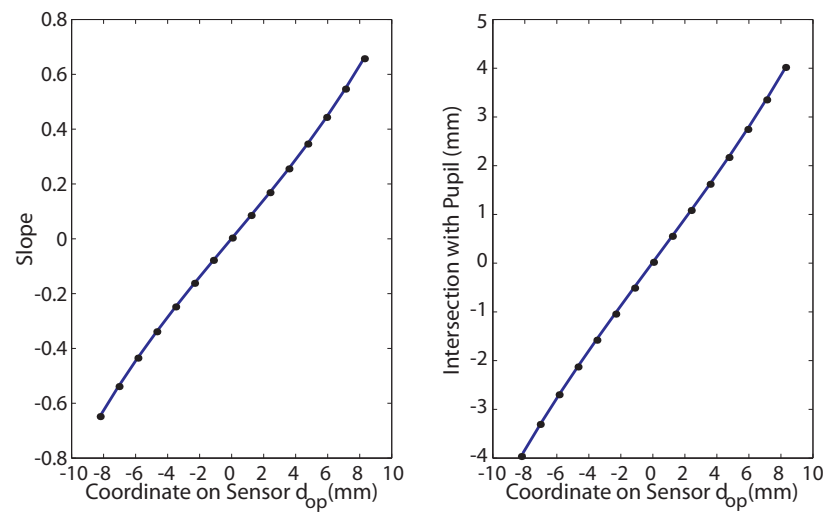

(b)

Fig. 4. Parametrization polynomials for the optical system of Fig. 3. (a) Isofocus surface parametrization: Fitted 2nd- and 3rd-order polynomials for the curvature and for the intersection with the optical axis. (b) Isopixel curve parametrization: Fitted 3rd-order polynomials for the line slope and for the intersection with the pupil.

surfaces. Therefore, we will assume that they are also conic surfaces that can be parametrized by their conic constant, curvature, and intersection with the optical axis. Since the isofocus surfaces correspond to a specific sensor position, their three parameters can also be expressed as functions of the sensor position. In Fig. 3 we show the simulated isofocus surfaces with solid lines and the fitted conic surfaces with dashed lines. The curvature and intersection with the optical axis parameters of the fitted surfaces are displayed in Fig. 4(a) as polynomial functions of the sensor position. For each parameter, we fit the least-order polynomial that captures its variability effectively. The conic constant need not vary (fixed at -0.5 ), because it was observed that the surface variation can be captured by the curvature successfully.

The isopixel curves are lines, and it is straightforward to parametrize them using their slope and their distance from the optical axis at the pupil. Each isopixel curve corresponds to one pixel on the image, and its parameters are functions of the pixel's offset (measured from the image center) due to the rotational symmetry of the system. For the $2 \mathrm{D}$ case, two parameters are required. In Fig. 3 the simulated isopixel curves are displayed with solid lines, and the fitted lines are dashed lines. The parameters of the fitted lines are shown in Fig. 4(b) as polynomial functions of the pixel's coordinate on the image sensor. For each parameter, we fit the least-order polynomial that captured its variability effectively.

As can be seen in Fig. 4 the parametrizing functions are indeed injections. As a result, intraocular localization can be unambiguous. Thus, a technique for 3D intraocular localization with a wide angle is possible.

\section{Localization in a Model Eye}

To demonstrate the effectiveness of the proposed wideangle localization method, we perform a full localization experiment. Firstly, we create an appropriate MVO based on design criteria from preliminary research [9]. Based on this design, we perform a localization-from-focus experiment using a human eye model.

\section{A. The Model Eye}

As an experimental testbed, we use the model eye [16] from Gwb International, Ltd. This eye is equipped with a plano-convex lens of $\sim 36 \mathrm{~mm}$ focal length that mimics the compound optical system of the human eye. The model eye contains no liquid, and thus, the lens can be used by itself. Gwb International, Ltd. disclosed the lens' parameters so that simulations can be performed accurately. The dimensions of the model eye were measured to estimate its retinal depth and shape.

The optical parameters of human eyes can also be measured. In [14], [15], techniques that allow the measurement of the intraocular lens parameters and the corneal topography for individual patients are presented. As a result, the parameters required for the optical modeling can be provided for individual patients. Additionally, in [17] the retinal shape is estimated in vivo from MRI scans. In the following, whenever calibration is needed, we only use information that can be acquired in vivo.

\section{B. The Mechatronic Vitreoretinal Ophthalmoscope}

The MVO is a device that consists of two components: a condensing lens that is kept at a constant position with 


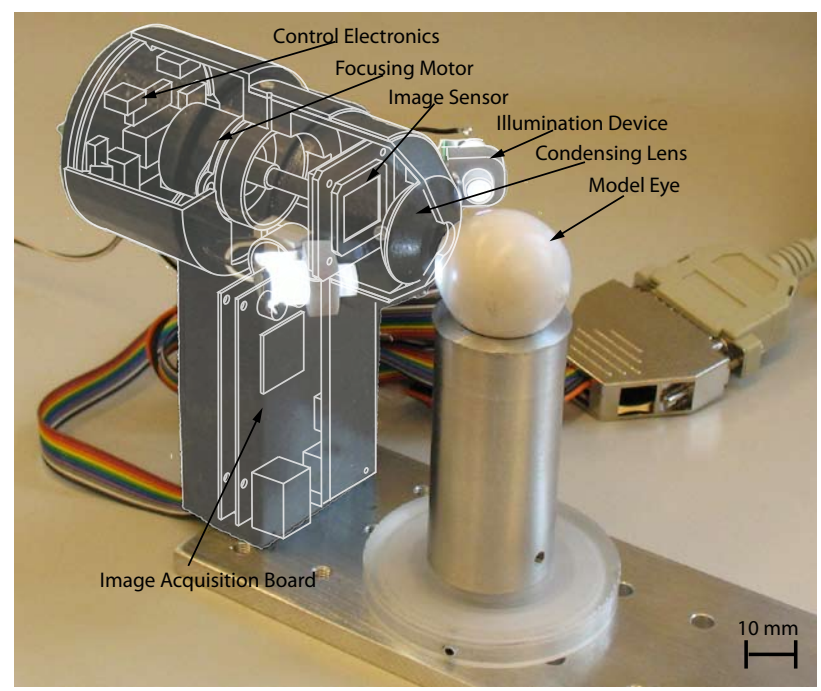

Fig. 5. CAD-design overlayed on the fabricated MVO prototype together with the model eye [16].

respect to the eye, and a sensor that captures the aerial image directly and moves with respect to the lens to focus on objects throughout the eye.

The condensing lens must be selected with respect to the specific application. For example, in [9] a high field-ofview was required and the Digital Wide Field ${ }^{\circledR}[18]$ from Volk Optical Inc. was used. In [19] high magnification was more important than super-wide field-of-view and the Digital High $\mathrm{Mag}^{\circledR}$ [20] lens was employed. The drawback in using commercial lenses is that their surface parameters are unknown. In this paper, since we require accurate modeling of the optical system, we use a custom-made condensing lens based on [13] (see Fig. 2(b) for parameters, where the refraction index $\mathrm{n}$ was changed to 1.531). This condensing lens causes a $0.78 \times$ magnification, thus, an object of $100 \mu \mathrm{m}$ near the retina would create an image of $78 \mu \mathrm{m}$.

The image sensor should be of adequate size in order to capture the full field-of-view, and it should have a shallow depth-of-field in order to be appropriate for focus-based localization applications. Moreover, it should be able to spatially resolve small structures sufficiently. The MVO is equipped with the board-level version of PL-B742U-BL from PixeLINK, which has a CMOS sensor with a $6.7 \mu \mathrm{m} \times 6.7 \mu \mathrm{m}$ sensing element, and $1280 \times 1024$ noninterpolated resolution. The pixel size guarantees both a small depth-of-field and adequate spatial resolution. This sensor cannot capture the full image of the retina, but can be used for working inside the validity area of the Navarro eye.

In order to obtain the desired localization resolution, the motion resolution of the motors moving the sensor should be sufficiently small. In the MVO, the focusing mechanism is based on the LP2515S0104-TR3x1 motors from Nanotec $\mathrm{GmbH}$ that have a motion resolution of $42 \mu \mathrm{m}$ per step. This resolution, based on the slope of the curve of Fig. 2(c), theoretically allows intraocular depth estimation with $100 \mu \mathrm{m}$ precision. In our experiments, we wanted to examine the effect of different motor steps in estimating the in-focus

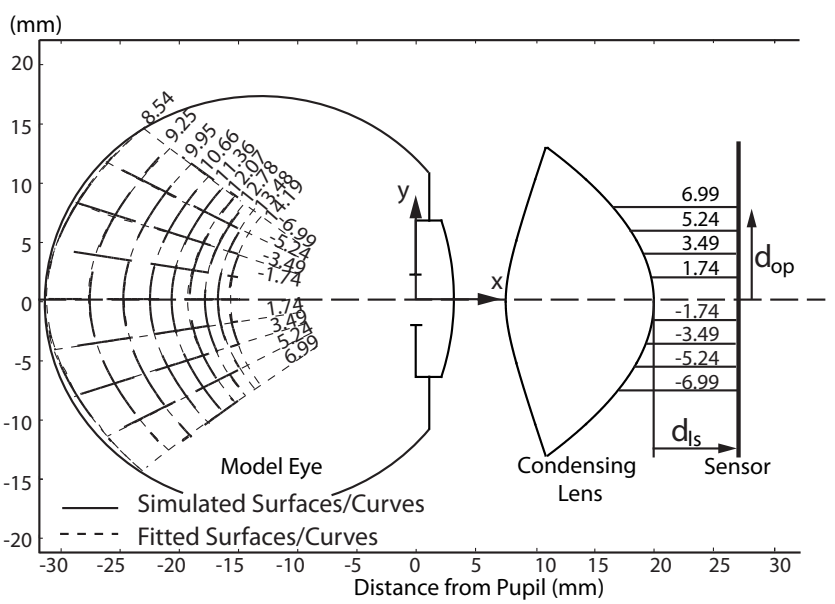

Fig. 6. Simulation of the isofocus surfaces and isopixel curves for the system composed of the model eye and the MVO. The different isofocus surfaces correspond to the distance from the lens to the sensor $\left(d_{l s}\right)$, for uniform sensor steps of $\sim 0.7 \mathrm{~mm}$. The isopixel curves correspond to pixel distances from the optical axis $\left(d_{o p}\right)$, for uniform steps of $\sim 1.75 \mathrm{~mm}$. The simulated surfaces and the fitted ones are hard to distinguish.

sensor position, so, we used a Sutter linear stage. Our final results are based on a motor resolution of $50 \mu \mathrm{m}$.

Another benefit of the MVO is that it minimizes the illumination necessary for adequate image quality. The MVO uses the fewest number of lenses required to perform intraocular imaging, and by minimizing the number of lens surfaces within the light path to two the light that must be exerted on the patient's retina is minimized. This minimal illumination requirement, in combination with the wide field-of-view, adequate spatial resolution, precise knowledge of its optical elements, and ability to obtain depth information from focus, makes the MVO promising for the localization of intraocular devices. The CAD design and the fabricated MVO can be seen in Fig. 5.

\section{The Isofocus Surfaces and Isopixel Curves}

The optical system under examination is composed of the model eye described in Sec. IV-A and the MVO described in Sec. IV-B. The simulated isofocus surfaces and isopixel curves of the composite system are shown in Fig. 6. Based on these simulations, we parametrize the isofocus surfaces (Fig. 7(a)) and the isopixel curves (Fig. 7(b)). The behavior of the parameters is similar to the one displayed in Fig. 4(a) and Fig. 4(b). The assumed conic constant of the isofocus surfaces is kept constant at -1.05 .

\section{Calibration}

Due to measurement and calculation uncertainties, the model and simulations are not entirely accurate. Thus, methods for calibrating the isofocus surfaces and isopixel curves of the actual system are required. Preliminary experiments show that calibration of the isopixel curves is not necessary because their relative impact on the localization accuracy is low.

In order to calibrate the isofocus surfaces for their intersection with the optical axis, we perform an on-optical-axis depth-from-focus experiment on the aligned optical system. 

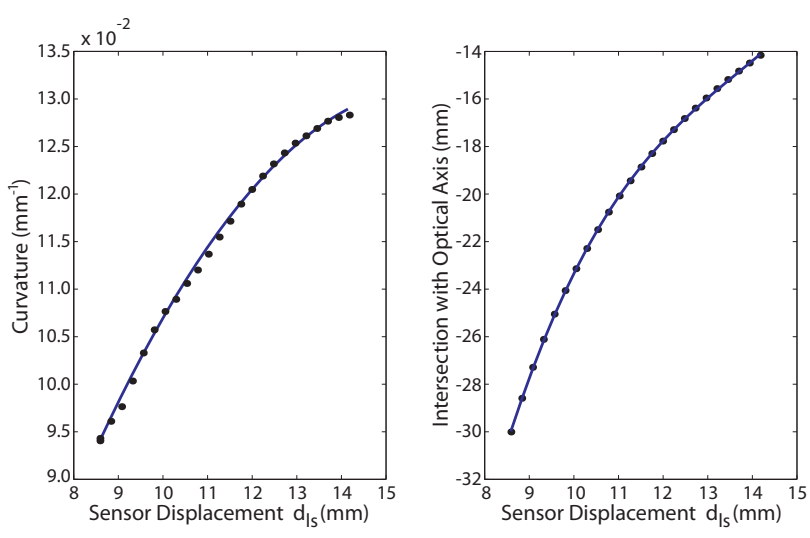

(a)
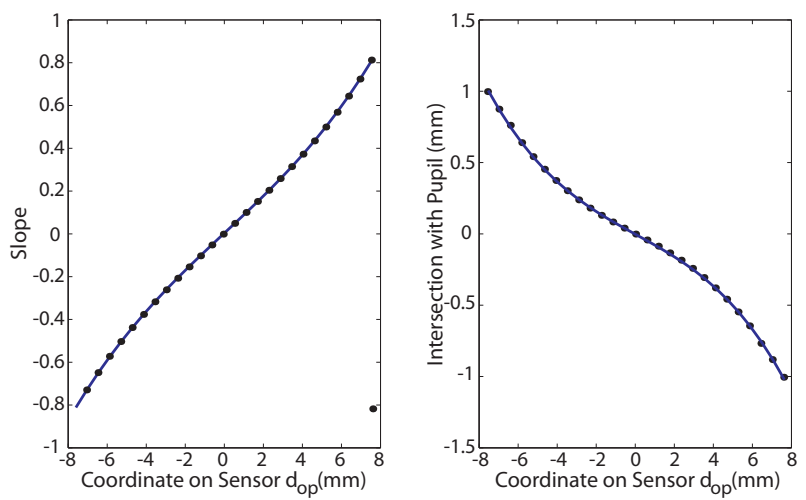

(b)

Fig. 7. (a) Isofocus surface parametrization: Fitted 2nd- and 3rd-order polynomials for the curvature and for the intersection with the optical axis. (b) Isopixel curve parametrization: Fitted 3rd-order polynomials for the slope and for the intersection with the pupil.

We use a Sutter linear micromanipulation stage to move a checkerboard calibration pattern in the model eye with $1 \mathrm{~mm}$ steps, and estimate the in-focus sensor position using the normalized variance of the captured image as a focus indicator. According to [21], the normalized variance is the most robust metric of image focusing for noisy images. The estimated in-focus sensor positions with respect to different depths in the model eye can be seen in Fig. 8. The uncalibrated model fit is displayed with a dotted line, and as can be seen, calibration is needed. This is the model extracted directly from the simulations (Fig. 7(a) (right)).

In the model eye, we can calibrate for the relationship between the in-focus sensor position and the depth of the object by using the full set of data points (Fig. 8). However, such an approach would be clinically invasive as it would require a vitrectomy and a moving device inside the eye. The only minimally invasive biometric data available are the depth and shape of the retina. Assuming that there are accumulated errors that can be lumped and included as errors in the estimated image and object positions, we show in [9] that by using a first-order model of the optics, calibration using only the depth of the retina is possible.

By adapting this method to our current framework, we are able to biometrically calibrate for the parameters of the polynomial that describes the intersection of the isofocus

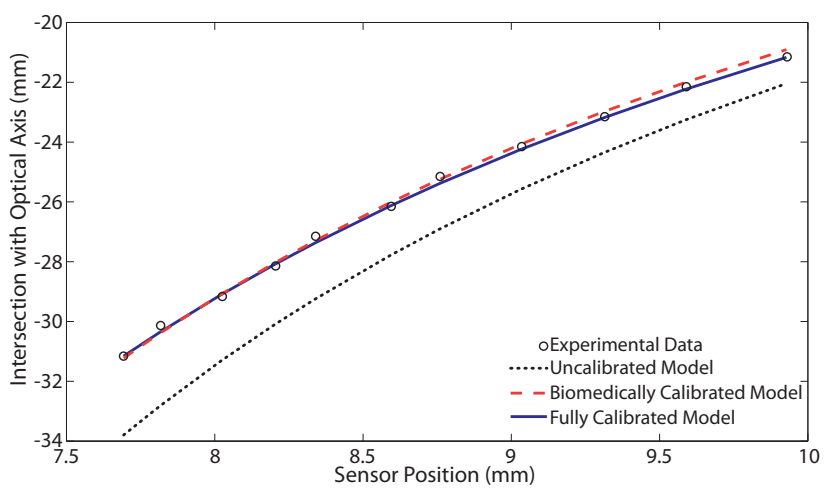

Fig. 8. Different model fits for the function describing the intersection of isofocus surfaces with the optical axis (measured from the pupil) with respect to the in-focus sensor position. Biometric calibration errors: $\max =$ $318 \mu \mathrm{m}$, mean $=159 \mu \mathrm{m}$, std $=94 \mu \mathrm{m}$.

surfaces with the optical axis. The resulting fit can be seen in Fig. 8. This technique allows calibration for one parameter of the isofocus surfaces.

The remaining two parameters of the isofocus surfaces control the shape of the isofocus surfaces but not their position. The condensing lens is designed to create a flat aerial image of the retinal surface, and our experiments have shown that we can use it to capture an overall sharp image of the model eye's retina. Therefore, we conclude that there exists an isofocus surface that corresponds to the retinal surface, and we consider it as the $1^{\text {st }}$ surface. From Fig. 6 we see that the $1^{\text {st }}$ isofocus surface does indeed roughly correspond to the retinal shape (mean error $=371 \mu \mathrm{m}$ ). As a result, calibration for the conic constant and the curvature is not needed. If our model was not accurately predicting the shape of the retina, then we would calibrate the parameters of the $1^{\text {st }}$ isofocus surface so that is has exactly the same shape as the retina.

\section{E. Results}

In order to estimate the validity of our wide-angle localization algorithm, we performed a localization experiment for various angles with respect to the optical axis, and various distances from the pupil. In Fig. 9, the results using the proposed wide-angle localization algorithm are displayed. Since the optical setup is well-controlled, the results are repeatable; inaccuracies are primarily not due to measurement noise. For comparison with existing work, we show the results based on the paraxial localization algorithm presented in [9] for angles up to $10^{\circ}$ from the optical axis. As expected, the results of paraxial localization deteriorate as the angles increase. However, the predictions of the new localization algorithm remain close to the actual values.

In Table I the localization errors with respect to increasing angles and distances from the pupil are shown. The rows show the errors for varying distances from the pupil, and the columns show the errors for varying angles at fixed distances. As the angles increase, the mean error and the standard deviation are stable. The proposed wide-angle localization 


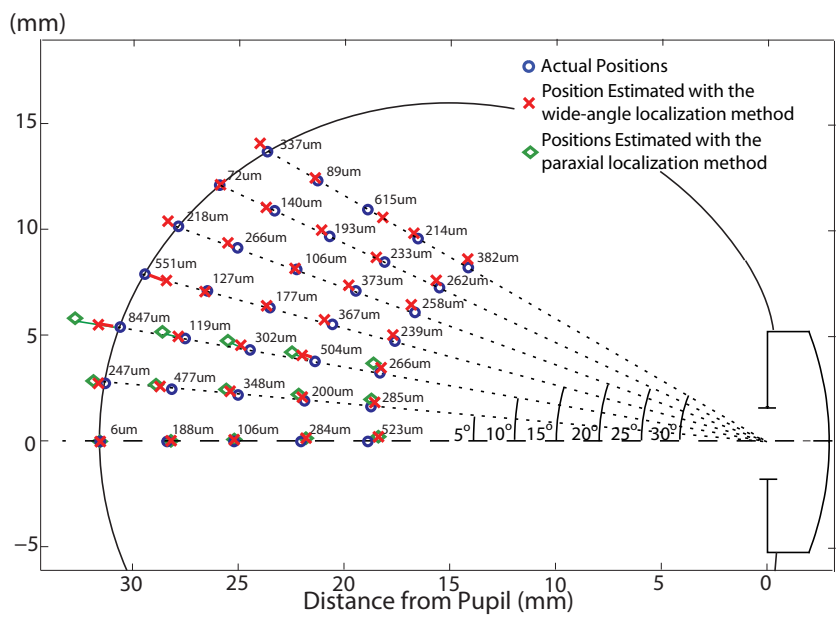

Fig. 9. Localization experiment showing the validity of the wide-angle localization algorithm. Experiment is shown for the condensing lens [13], and the model eye [16]. The paraxial model of [9], which loses accuracy away from the optical axis, is also shown for comparison.

TABLE I

INDIVIDUAL AND MEAN ERRORS FOR THE EXPERIMENT IN FIG. 9

\begin{tabular}{rrrrrr|cc} 
angles & \multicolumn{9}{c}{ errors $(\mu \mathrm{m})$} & \multicolumn{1}{c}{ mean } & std \\
\hline $0^{\circ}$ & 6 & 188 & 106 & 248 & 523 & 214 & 195 \\
$5^{\circ}$ & 247 & 477 & 348 & 200 & 285 & 311 & 107 \\
$10^{\circ}$ & 847 & 119 & 302 & 504 & 266 & 407 & 281 \\
$15^{\circ}$ & 551 & 127 & 177 & 367 & 239 & 292 & 170 \\
$20^{\circ}$ & 218 & 266 & 106 & 373 & 258 & 244 & 96 \\
$25^{\circ}$ & 72 & 140 & 193 & 223 & 262 & 178 & 74 \\
$30^{\circ}$ & 337 & 89 & 615 & 214 & 382 & 327 & 197 \\
\hline mean & 325 & 201 & 264 & 304 & 316 & & \\
std & 269 & 125 & 167 & 105 & 95 & & \\
\hline overall & & & & & & 282 & 173
\end{tabular}

method is successful and can be used for regions away from the optical axis with accuracy.

\section{Conclusions}

In this paper we presented the first wide-angle intraocular localization algorithm. Based on indirect ophthalmoscopy, the algorithm uses a condensing lens on an eye model in order to extract localization information from focus. The condensing lens can be custom made, and the parameters of the human eye can be measured [14], [15], [17] for individuals. We demonstrated successful localization through experiments in a model eye. We prototyped a mechatronic vitreoretinal ophthalmoscope that can be used for imaging and localizing intraocular agents in the posterior of the human eye. Moreover, we presented calibration techniques that rely only on measurements that could be obtained clinically. The localization results are very satisfactory.

In the future, we will evaluate the robustness of the presented approach with respect to variations in the illumination and uncertainties in the optical system. This is an important step towards in vivo intraocular localization.

\section{REFERENCES}

[1] B. Mitchell, J. Koo, I. Iordachita, P. Kazanzides, A. Kapoor, J. Handa, G. Hager, and R. Taylor, "Development and application of a new steady-hand manipulator for retinal surgery," IEEE Int. Conf. Robotics and Automation, pp. 623-629, 2007.

[2] C. Riviere, W. Ang, and P. Khosla, "Toward active tremor canceling in handheld microsurgical instruments," IEEE Trans. Robotics and Automation, vol. 19, no. 5, pp. 793-800, 2003.

[3] W. Wei, R. Goldman, N. Simaan, H. Fine, and S. Chang, "Design and theoretical evaluation of micro-surgical manipulators for orbital manipulation and intraocular dexterity," IEEE Int. Conf. Robotics and Automation, pp. 3389-3395, 2007.

[4] K. B. Yesin, K. Vollmers, and B. J. Nelson, "Modeling and control of untethered biomicrorobots in a fluidic environment using electromagnetic fields," Int. J. Robotics Research, vol. 25, no. 5-6, pp. 527-536, 2006.

[5] M. P. Snead, M. P. Rubinstein, and P. M. Jacobs, "The optics of fundus examination," Sur. Ophthalmology, vol. 36, no. 6, pp. 439-445, 1992.

[6] T. Drummond and R. Cipolla, "Real-time visual tracking of complex structures," IEEE Trans. Pattern Analysis and Machine Intelligence, pp. 932-946, 2002.

[7] Z. Nagy, O. Ergeneman, J. J. Abbott, M. Hutter, A. Hirt, and B. Nelson, "Modeling assembled-MEMS microrobots for wireless magnetic control," IEEE Int. Conf. Robotics and Automation, pp. 874-879, 2008

[8] J. J. Abbott, O. Ergeneman, M. P. Kummer, A. M. Hirt, and B. J. Nelson, "Modeling magnetic torque and force for controlled manipulation of soft-magnetic bodies," IEEE Trans. Robotics, vol. 23, no. 6, pp. 1247-1252, 2007.

[9] C. Bergeles, K. Shamaei, J. J. Abbott, and B. J. Nelson, "On imaging and localizing untethered intraocular devices with a stationary camera," IEEE Int. Conf. Biomedical Robotics and Biomechatronics, pp. 489-494, 2008.

[10] J. Ens and P. Lawrence, "An investigation of methods for determining depth from focus," IEEE Trans. Pattern Analysis and Machine Intelligence, vol. 15, no. 2, pp. 97-108, 1993.

[11] K. B. Yesin, K. Vollmers, and B. J. Nelson, "Guidance of magnetic intraocular microrobots by active defocused tracking," IEEE/RSJ Int. Conf. Intelligent Robots and Systems, vol. 4, pp. 3309-3314, 2004

[12] I. Escudero-Sanz and R. Navarro, "Off-axis aberrations of a wideangle schematic eye model," J. Optical Society of America, vol. 16 no. 8, pp. 1881-1891, 1999.

[13] D. A. Volk, "Indirect ophthalmoscopy lens for use with split lamp or other biomicroscope," Jan. 6 1998, U.S. Patent 5,706,073.

[14] Y. Mejia-Barbosa and D. Malacara-Hernandez, "A review of methods for measuring corneal topography," J. Optometry and Vision Science, vol. 78 , no. 4, pp. $240-53,2001$

[15] T. Kirschkamp, M. Dunne, and J. C. Barry, "Phakometric measurement of ocular surface radii of curvature, axial separations and alignment in relaxed and accommodated human eyes," J. Ophthalmic and Physiological Optics, vol. 24, no. 2, pp. 65-73, 2004.

[16] (2009) Model eye (2mm pupil) from GWB International, Ltd. [Online]. Available: http://www.gwbinternational.com/model_eye.htm

[17] D. A. Atchison, N. Pritchard, K. L. Schmid, D. H. Scott, C. E. Jones, and J. M. Pope, "Shape of the retinal surface in emmetropia and myopia," Investigative Ophthalmology \& Visual Science, vol. 46, no. 8 , pp. 2698-2707, 2005.

[18] (2009) Digital Wide Field ${ }^{\circledR}$ lens from Volk Optical Inc. [Online]. Available: http://www.volk.com/main/product_info.php?pName= digital- wide- field\&osCsid=cfb7fffe 1995c879f63871608e2f6bd4

[19] C. Bergeles, G. Fagogenis, J. J. Abbott, and B. J. Nelson, "Tracking intraocular microdevices based on colorspace evaluation and statistical color/shape information," IEEE Int. Conf. Robotics and Automation, 2009, to appear.

[20] (2009) Digital High $\operatorname{Mag}^{\circledR}$ lens from Volk Optical Inc. [Online]. Available: http://www.volk.com/main/product_info.php?pName= digital-high-mag\&osCsid=cfb7fffe 1995c879f63871608e2f6bd4

[21] Y. Sun, S. Duthaler, and B. J. Nelson, "Autofocusing in computer microscopy: selecting the optimal focus algorithm," J. Microscopy Research and Technique, vol. 65, no. 3, pp. 139-149, 2004. 